\title{
Evolution of binding preferences among whole-genome duplicated
}

\section{transcription factors}

\author{
Tamar Gera ${ }^{1,3}$, Felix Jonas ${ }^{1,3}$, Roye More $^{1}$, and Naama Barkai ${ }^{1 *}$ \\ ${ }^{1}$ Department of Molecular Genetics, Weizmann Institute of Science; Rehovot 76100, Israel. \\ ${ }^{3}$ Co-first authors \\ *Corresponding author. Email: naama.barkai@weizmann.ac.il
}




\begin{abstract}
Throughout evolution, new transcription factors (TFs) emerge by gene duplication, promoting growth and rewiring of transcriptional networks. How TF duplicates diverge is known for only a few studied cases. To provide a genome-scale view, we considered the $35 \%$ of budding yeast TFs, classified as whole-genome duplication (WGD)-retained paralogs. Using high-resolution profiling, we find that $\sim 60 \%$ of paralogs evolved differential binding preferences. We show that this divergence results primarily from variations outside the DNA binding domains (DBDs), while DBD preferences remain largely conserved. Analysis of non-WGD orthologs revealed that ancestral preferences are unevenly split between duplicates, while new targets are acquired preferentially by the least conserved paralog (biased sub/neo-functionalization). Dimer-forming paralogs evolved mostly one-sided dependency, while other paralogs interacted through lowmagnitude DNA-binding competition that minimized paralog interference. We discuss the implications of our findings for the evolutionary design of transcriptional networks.
\end{abstract}




\section{Main}

Transcription factors (TFs) bind at regulatory regions to activate or repress transcription. Cells express hundreds of TFs that together transit cells between different expression programs. Despite rapid advances, our understanding of transcriptional networks is still fragmented (1). For example, different TFs that bind to similar DNA sequences in-vitro (2-10) localize to different genomic sites in-vivo through mechanisms still poorly understood. Further, with increasing organism complexity, new TFs emerge, yet we know little about the means through which these emerging TFs adapt new targets and integrate into the existing transcriptional network.

Gene duplication is the major source of new TFs (11-15), with whole-genome duplications (WGDs) playing a particularly important role (16-26). In budding yeast, $\sim 35 \%$ of all TFs are associated with a single WGD event dating 100 million years ago (Fig. 1A) (19, 20). We reasoned that this set of TF duplicates, all generated at the same time and subjected to the same evolutionary history, provides a convenient platform for studying the fate of retained duplicated TF genes.

TF duplicates (paralogs) can diverge through changes in expression, regulation or function (2744). Of particular interest is the selection of in-vivo binding sites, as these define potential regulatory targets. Mechanisms driving such divergence include changes in co-factor binding (45) or in DNA motif preferences (46-51). The prevalence of these different scenarios is still unclear, since studied cases considered only a few paralogs, of different ages and origins, and divergence was studied at individual targets. We therefore aimed to provide a genome-scale view, by comparing genome-wide binding preferences among the full set of WGD TFs in budding yeast. 


\section{Results}

\section{Divergence of binding preferences among WGD TFS}

Within the Saccharomyces cerevisiae genome database (SGD) (52), 82 DNA binding domain (DBD)-containing proteins are classified as WGD-retained paralogs (Fig. 1A,B). We refined this list to include only pairs where both paralogs act as specific TFs (35 pairs, table S1), and defined the binding locations of these TFs across the genome using ChEC-seq (53). A total of 30 pairs (60 TFs) were successfully profiled, as verified by data reproducibility (Pearson's $r>0.95$ in promoter binding preferences, Fig. 1C,D) and manual literature survey (fig. S1). A large fraction of TFs were bound at their own or their paralog's promoters, potentially forming regulatory circuits (15) (Fig. 1E).

Perhaps unexpectedly, binding preferences were conserved (Pearson's $r>0.8$ ) among $\sim 40 \%$ of paralogs, most notably these of the $\mathrm{C} 2 \mathrm{H} 2$ zinc-finger family (6/10 pairs, e.g. Met31/Met32, Fig. 1C,D). Furthermore, most diverging paralogs still shared promoter targets (Fig. 1F). In some cases, the two duplicates localized to the same promoters but with different relative strengths (e.g. Gzf3/Dal80, Fig. 1C). In other cases, some promoters were bound by both paralogs, while others by just one (e.g. Ace2/Swi5, Fig. 1C). Therefore, binding preferences diverge at a rate that differs between pairs, and, within each pair, differs between individual promoters.

\section{Paralog diverge through variations outside their DBDs}

TFs localize to genomic sites containing short motif sequences bound by their DBDs. The in-vivo binding could therefore diverge through DBD variations that modify motif preferences. To compare DBD sequences among paralogs, we aligned each pair and classified residues into these 
defining the $\mathrm{DBD}$ family (e.g. $\mathrm{C} / \mathrm{H}$ residues in the $\mathrm{C} 2 \mathrm{H} 2$ zinc-finger domains), those that contribute to DNA motif preference (54), and the remaining ones (Fig. 2A,B and fig. S2).

Sequence conservation varied between DBD families (Fig. 2B). In particular, specificityconferring residues often varied between paralogs of the fungal-specific zinc-cluster family (Fig. 2B), but remained invariant between paralogs of the $\mathrm{C} 2 \mathrm{H} 2$ zinc-finger family and, to a lesser extent, in other families (e.g. Rph1/Gis1, Fig. 2A,B). Examining motif preferences derived from in-vitro data, we noted that reported preferences $(2,54)$ are often (although not always) similar amongst paralogs (Fig. 2A and fig. S2).

DBD variations may therefore contribute to the divergence of zinc-cluster paralogs, but appear to play a lesser role in paralogs of the $\mathrm{C} 2 \mathrm{H} 2$ zinc finger and perhaps other families. To test this, we swapped DBDs between paralogs (Fig. 2C), reasoning that if DBD variations drive divergence, swapping DBDs would swap promoter preferences. Conversely, if critical variations are located outside the DBD, swapping will be of little effect. Consistent with our sequence analysis, DBD swapping perturbed binding for three of the four zinc-cluster TFs tested, although in none of these cases was DBD swapping sufficient for swapping promoter preferences (Fig. 2D,E). The fourth TF (Yrr1) remained largely invariant (Pearson's $r>0.8$ ) to DBD swapping, as did the eleven additionally tested TFs, taken from six different families. Of note, this invariance to DBD swapping characterizing most TFs was observed not only when comparing promoter preferences, but also when comparing in-vivo preferences to DNA 7-mers (Fig. 2D and fig. S2). We conclude that, for most duplicate pairs, the variations driving divergence in promoter binding preferences are located outside the DBDs. 


\section{Dependencies and competitions between TF paralogs}

Evolved interactions between paralogs could affect binding preferences. TFs that bind DNA as dimers, for example, may bind as heterodimers to a subset of sites. Paralogs may also compete for binding, either directly or by interacting with a shared co-factor. In the broader context, cooperative interactions, where a TF depends on its paralog, may increase mutation fragility whereas binding competition, which allows a TF to access its perturbed paralog's sites, may increase mutation robustness (Fig. 3A). Both effects were observed in the context of proteinprotein interactions (44).

To test the prevalence of cooperative or competitive interactions, we measured TF binding upon paralog deletion, testing 55/60 TFs in our dataset (Fig. 3B and fig. S3). Two TFs completely lost binding signals (Pip2, Hms2), and additional two lost binding to their respective paralogs' targets (Da180, Tbs1). These large-scale effects, however, were infrequent (Fig. 3B,C). Cooperative interactions were generally minor (e.g. Stp2), as were compensatory interactions (e.g. Pdr3 or Ecm22; Fig. 3C,D). Therefore, strong interactions between TFs paralogs are rare and existing ones tend to increase mutation fragility.

\section{Classifying paralogs' evolutionary paths by analyzing non-WGD orthologs}

Two prevailing models explain paralog divergence (55-63): In the first, ancestral functions split between duplicates (sub-functionalization) while, in the second, one duplicate retains ancestral functions, while the second adapts a new role (neo-functionalization, Fig. 4A). As a first approach to distinguish these two scenarios, we used phylogenetic sequence analysis to compare the evolutionary rates of the regions outside the conserved DBD (Fig. 4B). This analysis is informative, since a neo-functionalizing paralog undergoes a period of relaxed selection, followed by rapid evolution, and therefore evolves at an accelerated rate $(64,65)$. Diverging paralogs of the 
$\mathrm{C} 2 \mathrm{H} 2$ zinc-finger family evolved symmetrically, at rates that did not distinguish between paralogs, consistent with sub-functionalization. By contrast, diverging paralogs of other families evolved asymmetrically, suggesting dominant neo-functionalization (Fig. 4B).

To test experimentally for sub- and neo-functionalization, we compared binding preferences of paralogs in our set to that of a corresponding non-WGD ortholog (Kluyveromyces lactis TF, expressed within S. cerevisiae; Fig. 4C). We reasoned that, in terms of binding preferences, this non-WGD ortholog might serve as a good proxy for the ancestor $(15,66,67)$. This was the case in pairs with clear expectations: binding of the $K$. lactis ortholog of Ixr1/Abf2 was indistinguishable from that of Ixr1 (Fig. 4C), consistent with Abf2 adopting a mitochondria role (68) (note Abf2's accelerated sequence evolution, Fig. 4B). Similarly, K. lactis orthologs of non-diverging duplicates retained highly similar binding preferences (Rsf2/Tda9 Fig. 4D and fig. S4 for additional pairs). Together, these results support the use of non-WGD orthologs to approximate ancestral preferences.

We next extended the analysis to divergent duplicates. We observed cases of clear sub- and neofunctionalization (Rph1/Gis1 and Vhr1/Vhr2, respectively Fig. 4D,E), but most pairs showed a combination of the two scenarios. Of note, in all 11 diverging cases, at least one paralog was more similar to the ortholog than to the other S. cerevisiae paralog and in 9 of them, the conserved paralog was also slower to evolve (Fig. 4E). Together, these results suggest that the prevalent route for diversification is through biased neo/sub-functionalization: ancestral preferences split unevenly between the duplicates, coupled to biased acquisition of novel targets (Fig. 4F and fig. S4). 


\section{Divergence of zinc-cluster paralogs: motif preferences and paralog interactions}

To gain molecular insights into the divergence of specific duplicates, we examined individual pairs, focusing first on the fungal-specific zinc-cluster family. TFs of this family bind DNA as dimers, recognizing a composite DNA motif that includes two spaced CGG sites (69). Binding specificity depends on the orientation of the DBD-bound CGG triplets, and the spacer length, which may depend on an unstructured linker flanking the DBD (Fig. 5A). As described above, when compared to other families, zinc-cluster paralogs showed a greater dependence on DBD variations (cf. Fig. 2) and were more likely to interact (cf. Fig. 3).

We searched for CGG-containing motifs in regions bound by the zinc-cluster TFs in our dataset. In three of the five diverging pairs, differences in binding preferences correlated with differential motif preferences (Fig. 5A: differential spacing/orientation - Ha19/Tbs1 and Oaf1/Pip2 or variation within the CGG - Pdr1/Pdr3). All three pairs interacted: Ha19/Tbs1 recruited each other to their preferred sites, likely through heterodimerization, and Pip2 localized exclusively to a subset of Oafl-preferred targets in an Oafl-dependent manner, consistent with obligatory heterodimerization (70). Notably, binding preferences of both heterodimers correlated well with that of the K. lactis ortholog. In the case of Pdr1/3, interaction took the form of competition, with Pdr1 outcompeting Pdr3 from accessing its preferred motif.

In these three cases described above, binding preferences evolved through a combination of DNAmotif preferences and protein-protein interactions. Contrasting these, CGG-containing motifs at Yrr1/Pdr8- or Upc2/Ecm22-bound sites did not distinguish between paralogs (Fig. 5A,C and fig. S5). Rather, paralogs localized to the same motifs, but within different promoters. This divergence of promoter preferences was largely DBD-independent in the case of Yrr1/Pdr8, but greatly benefitted from DBD-dependent competition in the case of Upc2/Ecm22 (Fig. 5C). In fact, upon 
UPC2 deletion or DBD swapping, Ecm22 gained access to strong Upc2 sites (Fig. 5C; note correspondence to $K$. lactis sites). Of note, while DBD-swapping retained binding at the TFspecific sites, it also allowed increased access to non-specific sites, suggesting co-evolution of the DBD and the linker domain, both of which varied substantially between the paralogs. We conclude that zinc-cluster paralogs evolved largely, but not exclusively, through changes in motif preferences or affinity, resulting from combined effects of variations within, and outside, their DBDs.

\section{Resolution of paralog interference through competitive binding}

Our analysis above described plausible evolutionary scenarios governing zinc-cluster paralog divergence, where motif preferences appear to play an important role. In most other paralogs, changes in motif preference, if exist, appear secondary to the major effective variations located outside the DBD. Still, even in such cases, DBD variations may play a role in resolving paralog interference $(45,71)$. Thus, since the two paralogs are initially redundant, divergence of binding preferences requires not only the acquisition of differential preferences, but also limiting residual, and possibly interfering paralog's cross binding. In the case of the non-WGD pair Mcm1/Arg80, for example, such interference was resolved by weakening direct Arg80-DNA binding $(45,71)$. We asked whether similar effects can be observed for WGD paralogs.

DBD variations contributed little to the divergence of Rph1/Gis1, the most diverged $\mathrm{C} 2 \mathrm{H} 2$ zincfinger paralogs. We noted, however, that Rph1 gained residual access to Gis1-binding sites upon GIS1 deletion or DBD swapping (Fig. 6A,B), and Rph1-exclusive targets contained a specific variant of the common Gis1/Rph1 motif, lacking from Gis1 binding sites (Fig. 6C,D). Phylogenetic analysis revealed two variations between the paralogs: first, Gis1 has lost a conserved demethylase activity (72), an event that occurred quite early following WGD (Fig. 6E and fig. S6). Second, 
Rph1's DBD experienced a more recent (minor) variation altering a conserved residue. This suggests the following evolutionary scenario: first, a loss of demethylase activity allowed Gis1 to specialize towards a subset of (weaker) ancestral targets and acquire new sites, through a primarily DBD-independent evolution. Second, mutations within Rph1's DBD prevented its binding to Gis1-specialized sites, thereby reducing paralog interference (Fig. 6E).

Finally, extending the analysis to other diverging paralogs suggested additional cases conforming to this same design showing limited binding competition (Fig. 6F and fig. S6). Together, these results suggest a common path, whereby DBD-independent divergence is complemented by asymmetric competition, limiting residual paralog interference.

\section{Discussion}

The binding of TFs at individual regulatory regions can vary through mutations that alter the DNA sequence or mutations that change TF binding preferences. While promoter mutations are gene specific, changes in TF binding preferences will affect multiple genes, and are therefore less likely to occur $(15,66,67)$. Evolution of binding preferences, however, is possible through $\mathrm{TF}$ duplication, which generates two redundant copies and relaxes selection. The evolutionary paths through which TF paralogs diverge their binding preferences may therefore hold the key to understanding the principles through which TFs select their binding locations across the genomes.

Studying a comprehensive set of WGD-retained TF paralogs, we found that the majority of pairs still share a large fraction of overlapping targets. In fact, even diverged paralogs still localized to common targets, although at different relative strengths. This gradual divergence was not explained by variations in the DBDs. In fact, we presented evidence that, with the exception of the zinc-cluster family, variations within the DBD contributed little to binding divergence. DBD preferences may play a minor role in setting promoter binding preferences but are primarily 
important for stabilizing binding within selected promoters. Further, cooperation and competition act to adjust binding profiles and limit paralog interference but, with few exceptions, are not the major factors guiding divergence. In this context, gradual, and promoter-specific divergence is harder to explain within prevailing models for TF specificity, but may be more consistent with the paradigm of distributed specificity we recently proposed $(1,73)$.

At the more global level, since duplication is the major means through which new TFs emerge, the evolutionary trajectories of retained paralogs shape the transcriptional network's design. Duplicates that diverge through sub-functionalization, for example, will confer a hierarchical design, where regulatory modules are gradually refined as the network expands. By contrast, neofunctionalization may support a distributed design, where new regulatory modules can emerge largely independent of previous connectivity. Our results reveal that neo-functionalization is quite common, although it is often combined with a biased sub-functionalization, namely uneven splitting of ancestral targets.

Finally, we focused our analysis on diverging paralogs, but it is notable that a significant fraction of paralog pairs $(\sim 40 \%)$ still binds at practically identical sites. Retention of these paralogs is therefore due to other properties. Duplicates' tendency to cross-bind their own promoters may suggest that interactions between duplicates have evolved to confer beneficial dynamic properties not achieved by a single TF $(15,74,75)$. Further studies are required to examine the potential advantages provided by such circuit-forming duplicates. 


\section{References and Notes}

1. T. Jana, S. Brodsky, N. Barkai, Speed-Specificity Trade-Offs in the Transcription Factors Search for Their Genomic Binding Sites. Trends Genet 37, 421-432 (2021).

2. Matthew T. Weirauch et al., Determination and Inference of Eukaryotic Transcription Factor Sequence Specificity. Cell 158, 1431-1443 (2014).

3. G.-H. Wei et al., Genome-wide analysis of ETS-family DNA-binding in vitro and in vivo. The EMBO Journal 29, 2147-2160 (2010).

4. A. Jolma et al., DNA-Binding Specificities of Human Transcription Factors. Cell 152, $327-$ 339 (2013).

5. V. Matys et al., TRANSFAC $\AA$ and its module TRANSCompel ${ }^{\circledR}$ : transcriptional gene regulation in eukaryotes. Nucleic Acids Research 34, D108-D110 (2006).

6. T. Siggers, J. Reddy, B. Barron, M. L. Bulyk, Diversification of transcription factor paralogs via noncanonical modularity in $\mathrm{C} 2 \mathrm{H} 2$ zinc finger DNA binding. Mol Cell 55, 640648 (2014).

7. S. Nakagawa, S. S. Gisselbrecht, J. M. Rogers, D. L. Hartl, M. L. Bulyk, DNA-binding specificity changes in the evolution of forkhead transcription factors. Proc Natl Acad Sci $U S A$ 110, 12349-12354 (2013).

8. J. M. Rogers, M. L. Bulyk, Diversification of transcription factor-DNA interactions and the evolution of gene regulatory networks. Wiley Interdiscip Rev Syst Biol Med, e1423 (2018).

9. M. F. Berger et al., Variation in homeodomain DNA binding revealed by high-resolution analysis of sequence preferences. Cell 133, 1266-1276 (2008).

10. N. Shen et al., Divergence in DNA Specificity among Paralogous Transcription Factors Contributes to Their Differential In Vivo Binding. Cell systems 6, 470-483.e478 (2018).

11. S. Ohno, U. Wolf, N. B. Atkin, Evolution from fish to mammals by gene duplication. Hereditas 59, 169-187 (1968).

12. A. Rosanova, A. Colliva, M. Osella, M. Caselle, Modelling the evolution of transcription factor binding preferences in complex eukaryotes. Sci Rep 7, 7596 (2017).

13. J. M. Vaquerizas, S. K. Kummerfeld, S. A. Teichmann, N. M. Luscombe, A census of human transcription factors: function, expression and evolution. Nature Reviews Genetics 10, 252-263 (2009).

14. M. Levine, R. Tjian, Transcription regulation and animal diversity. Nature 424, 147-151 (2003).

15. S. A. Teichmann, M. M. Babu, Gene regulatory network growth by duplication. Nat Genet 36, 492-496 (2004).

16. G. Blanc, K. H. Wolfe, Functional Divergence of Duplicated Genes Formed by Polyploidy during Arabidopsis Evolution. The Plant Cell 16, 1679-1691 (2004). 
17. S. Maere et al., Modeling gene and genome duplications in eukaryotes. Proc Natl Acad Sci $U S A$ 102, 5454-5459 (2005).

18. H. V. S. Rody, G. J. Baute, L. H. Rieseberg, L. O. Oliveira, Both mechanism and age of duplications contribute to biased gene retention patterns in plants. BMC genomics 18, 4646 (2017).

19. K. H. Wolfe, D. C. Shields, Molecular evidence for an ancient duplication of the entire yeast genome. Nature 387, 708-713 (1997).

20. M. Marcet-Houben, T. Gabaldón, Beyond the whole-genome duplication: phylogenetic evidence for an ancient interspecies hybridization in the baker's yeast lineage. PLoS Biol 13, e1002220 (2015).

21. J. A. Birchler, N. C. Riddle, D. L. Auger, R. A. Veitia, Dosage balance in gene regulation: biological implications. Trends Genet 21, 219-226 (2005).

22. M. Freeling, B. C. Thomas, Gene-balanced duplications, like tetraploidy, provide predictable drive to increase morphological complexity. Genome Res 16, 805-814 (2006).

23. P. P. Edger, J. C. Pires, Gene and genome duplications: the impact of dosage-sensitivity on the fate of nuclear genes. Chromosome Res 17, 699-717 (2009).

24. L. G. Lundin, Evolution of the vertebrate genome as reflected in paralogous chromosomal regions in man and the house mouse. Genomics 16, 1-19 (1993).

25. P. Dehal, J. L. Boore, Two rounds of whole genome duplication in the ancestral vertebrate. PLoS Biol 3, e314 (2005).

26. T. Blomme et al., The gain and loss of genes during 600 million years of vertebrate evolution. Genome Biol 7, R43 (2006).

27. T. Vavouri, J. I. Semple, B. Lehner, Widespread conservation of genetic redundancy during a billion years of eukaryotic evolution. Trends Genet 24, 485-488 (2008).

28. A. DeLuna et al., Exposing the fitness contribution of duplicated genes. Nat Genet 40, 676681 (2008).

29. G. Diss, D. Ascencio, A. DeLuna, C. R. Landry, Molecular mechanisms of paralogous compensation and the robustness of cellular networks. J Exp Zool B Mol Dev Evol 322, 488-499 (2014).

30. R. Kafri, M. Levy, Y. Pilpel, The regulatory utilization of genetic redundancy through responsive backup circuits. Proceedings of the National Academy of Sciences 103, 1165311658 (2006).

31. I. Wapinski, A. Pfeffer, N. Friedman, A. Regev, Natural history and evolutionary principles of gene duplication in fungi. Nature 449, 54-61 (2007).

32. Z. Gu et al., Role of duplicate genes in genetic robustness against null mutations. Nature 421, 63-66 (2003).

33. B. VanderSluis et al., Genetic interactions reveal the evolutionary trajectories of duplicate genes. Molecular systems biology 6, 429-429 (2010). 
34. V. Charoensawan, D. Wilson, S. A. Teichmann, Genomic repertoires of DNA-binding transcription factors across the tree of life. Nucleic Acids Research 38, 7364-7377 (2010).

35. D. L. Des Marais, M. D. Rausher, Escape from adaptive conflict after duplication in an anthocyanin pathway gene. Nature 454, 762-765 (2008).

36. T. L. Hsiao, D. Vitkup, Role of duplicate genes in robustness against deleterious human mutations. PLoS Genet 4, e1000014 (2008).

37. J. Ihmels, S. R. Collins, M. Schuldiner, N. J. Krogan, J. S. Weissman, Backup without redundancy: genetic interactions reveal the cost of duplicate gene loss. Mol Syst Biol 3, 86 (2007).

38. E. Kuzmin et al., Exploring whole-genome duplicate gene retention with complex genetic interaction analysis. Science 368, eaaz5667 (2020).

39. I. M. Ehrenreich, Evolution after genome duplication. Science 368, 1424-1425 (2020).

40. J. L. Payne, A. Wagner, Mechanisms of mutational robustness in transcriptional regulation. Front Genet 6, 322 (2015).

41. W.-H. Chen, X.-M. Zhao, V. van Noort, P. Bork, Human Monogenic Disease Genes Have Frequently Functionally Redundant Paralogs. PLOS Computational Biology 9, e1003073 (2013).

42. A. Burga, M. O. Casanueva, B. Lehner, Predicting mutation outcome from early stochastic variation in genetic interaction partners. Nature 480, 250-253 (2011).

43. L. T. MacNeil, A. J. M. Walhout, Gene regulatory networks and the role of robustness and stochasticity in the control of gene expression. Genome Research 21, 645-657 (2011).

44. G. Diss et al., Gene duplication can impart fragility, not robustness, in the yeast protein interaction network. Science 355, 630-634 (2017).

45. C. R. Baker, V. Hanson-Smith, A. D. Johnson, Following Gene Duplication, Paralog Interference Constrains Transcriptional Circuit Evolution. Science 342, 104-108 (2013).

46. J. C. Perez et al., How duplicated transcription regulators can diversify to govern the expression of nonoverlapping sets of genes. Genes Dev 28, 1272-1277 (2014).

47. Victoria M. Blake, S. Barolo, Genome Evolution: How Sister Genes Grow Apart. Current Biology 24, R695-R697 (2014).

48. A. N. McKeown et al., Evolution of DNA specificity in a transcription factor family produced a new gene regulatory module. Cell 159, 58-68 (2014).

49. J. T. Bridgham, J. E. Brown, A. Rodríguez-Marí, J. M. Catchen, J. W. Thornton, Evolution of a New Function by Degenerative Mutation in Cephalochordate Steroid Receptors. PLOS Genetics 4, e1000191 (2008).

50. M. V. Humbert, R. M. Rasia, S. K. Checa, F. C. Soncini, Protein signatures that promote operator selectivity among paralog MerR monovalent metal ion regulators. $\mathrm{J}$ Biol Chem 288, 20510-20519 (2013).

51. K. Pougach et al., Duplication of a promiscuous transcription factor drives the emergence of a new regulatory network. Nature Communications 5, 4868 (2014). 
52. J. M. Cherry et al., Saccharomyces Genome Database: the genomics resource of budding yeast. Nucleic Acids Res 40, D700-705 (2012).

53. G. E. Zentner, S. Kasinathan, B. Xin, R. Rohs, S. Henikoff, ChEC-seq kinetics discriminates transcription factor binding sites by DNA sequence and shape in vivo. Nature Communications 6, 8733 (2015).

54. S. A. Lambert et al., Similarity regression predicts evolution of transcription factor sequence specificity. Nat Genet 51, 981-989 (2019).

55. K. Voordeckers et al., Reconstruction of Ancestral Metabolic Enzymes Reveals Molecular Mechanisms Underlying Evolutionary Innovation through Gene Duplication. PLOS Biology 10, e1001446 (2012).

56. M. Lynch, J. S. Conery, The Evolutionary Fate and Consequences of Duplicate Genes. Science 290, 1151-1155 (2000).

57. R. P. Sugino, H. Innan, Selection for more of the same product as a force to enhance concerted evolution of duplicated genes. Trends in Genetics 22, 642-644 (2006).

58. M. Lynch, A. Force, The probability of duplicate gene preservation by subfunctionalization. Genetics 154, 459-473 (2000).

59. G. C. Conant, K. H. Wolfe, Functional Partitioning of Yeast Co-Expression Networks after Genome Duplication. PLOS Biology 4, e109 (2006).

60. A. Force et al., Preservation of Duplicate Genes by Complementary, Degenerative Mutations. Genetics 151, 1531-1545 (1999).

61. F. A. Kondrashov, I. B. Rogozin, Y. I. Wolf, E. V. Koonin, Selection in the evolution of gene duplications. Genome Biol 3, Research0008 (2002).

62. X. He, J. Zhang, Rapid Subfunctionalization Accompanied by Prolonged and Substantial Neofunctionalization in Duplicate Gene Evolution. Genetics 169, 1157-1164 (2005).

63. K. Nowick, L. Stubbs, Lineage-specific transcription factors and the evolution of gene regulatory networks. Brief Funct Genomics 9, 65-78 (2010).

64. C. Pegueroles, S. Laurie, M. M. Albà, Accelerated Evolution after Gene Duplication: A Time-Dependent Process Affecting Just One Copy. Molecular Biology and Evolution 30, 1830-1842 (2013).

65. K. P. Byrne, K. H. Wolfe, Consistent Patterns of Rate Asymmetry and Gene Loss Indicate Widespread Neofunctionalization of Yeast Genes After Whole-Genome Duplication. Genetics 175, 1341-1350 (2007).

66. C. C. Hsia, W. McGinnis, Evolution of transcription factor function. Current Opinion in Genetics \& Development 13, 199-206 (2003).

67. S. B. Carroll, Evolution at Two Levels: On Genes and Form. PLOS Biology 3, e245 (2005).

68. F. Caron, C. Jacq, J. Rouvière-Yaniv, Characterization of a histone-like protein extracted from yeast mitochondria. Proc Natl Acad Sci U S A 76, 4265-4269 (1979). 
69. S. MacPherson, M. Larochelle, B. Turcotte, A fungal family of transcriptional regulators: the zinc cluster proteins. Microbiology and molecular biology reviews : MMBR 70, 583604 (2006).

70. H. Rottensteiner, A. J. Kal, B. Hamilton, H. Ruis, H. F. Tabak, A heterodimer of the Zn2Cys6 transcription factors Pip2p and Oaflp controls induction of genes encoding peroxisomal proteins in Saccharomyces cerevisiae. Eur J Biochem 247, 776-783 (1997).

71. E. Kaltenegger, D. Ober, Paralogue Interference Affects the Dynamics after Gene Duplication. Trends in Plant Science 20, 814-821 (2015).

72. R. J. Klose, E. M. Kallin, Y. Zhang, JmjC-domain-containing proteins and histone demethylation. Nature Reviews Genetics 7, 715-727 (2006).

73. B. S et al., Intrinsically Disordered Regions Direct Transcription Factor In Vivo Binding Specificity. Molecular cell 79, (2020).

74. M. Chapal, S. Mintzer, S. Brodsky, M. Carmi, N. Barkai, Resolving noise-control conflict by gene duplication. PLOS Biology 17, e3000289 (2019).

75. B. Lehner, Conflict between noise and plasticity in yeast. PLoS Genet 6, e1001185 (2010).

76. B. Mészáros, G. Erdos, Z. Dosztányi, IUPred2A: context-dependent prediction of protein disorder as a function of redox state and protein binding. Nucleic Acids Res 46, W329W337 (2018).

77. R. a. M. G. a. H. J. Anand, Cas9-mediated gene editing in Saccharomyces cerevisiae. Protocol Exchange, (2017).

78. K. Labun et al., CHOPCHOP v3: expanding the CRISPR web toolbox beyond genome editing. Nucleic Acids Research 47, (2021).

79. B. CB et al., Designer deletion strains derived from Saccharomyces cerevisiae S288C: a useful set of strains and plasmids for PCR-mediated gene disruption and other applications. Yeast (Chichester, England) 14, (1998).

80. G. RD, S. RH, W. AR, W. RA, Studies on the transformation of intact yeast cells by the LiAc/SS-DNA/PEG procedure. Yeast (Chichester, England) 11, (1995).

81. M. Meurer et al., Genome-wide C-SWAT library for high-throughput yeast genome tagging. Nat Methods 15, 598-600 (2018).

82. U. Weill et al., Genome-wide SWAp-Tag yeast libraries for proteome exploration. Nat Methods 15, 617-622 (2018).

83. O. Lupo et al., Accumulation of cis- and trans-regulatory variations is associated with phenotypic divergence of a complex trait between yeast species. G3 Genes $\mid$ Genomes $\mid$ Genetics 11, (2021).

84. H. DW, D. TN, M. EG, Fluorescence resonance energy transfer using color variants of green fluorescent protein. Methods in enzymology 351, (2002).

85. C. Janke et al., A versatile toolbox for PCR-based tagging of yeast genes: new fluorescent proteins, more markers and promoter substitution cassettes. Yeast 21, 947-962 (2004). 
86. P. J. Skene, S. Henikoff, An efficient targeted nuclease strategy for high-resolution mapping of DNA binding sites. Elife 6, (2017).

87. R. Blecher-Gonen et al., High-throughput chromatin immunoprecipitation for genomewide mapping of in vivo protein-DNA interactions and epigenomic states. Nature Protocols 8, 539-554 (2013).

88. M. Martin, Cutadapt removes adapter sequences from high-throughput sequencing reads. EMBnet J 17, 10 (2011).

89. A. R. Quinlan, I. M. Hall, BEDTools: a flexible suite of utilities for comparing genomic features. Bioinformatics 26, 841-842 (2010).

90. D. Park, A. R. Morris, A. Battenhouse, V. R. Iyer, Simultaneous mapping of transcript ends at single-nucleotide resolution and identification of widespread promoter-associated noncoding RNA governed by TATA elements. Nucleic Acids Res 42, 3736-3749 (2014).

91. V. Pelechano, W. Wei, L. M. Steinmetz, Extensive transcriptional heterogeneity revealed by isoform profiling. Nature 497, 127-131 (2013).

92. R. A. Policastro, R. T. Raborn, V. P. Brendel, G. E. Zentner, Simple and efficient profiling of transcription initiation and transcript levels with STRIPE-seq. Genome Res 30, 910-923 (2020).

93. R. V. Chereji, S. Ramachandran, T. D. Bryson, S. Henikoff, Precise genome-wide mapping of single nucleosomes and linkers in vivo. Genome Biol 19, 19 (2018).

94. J. Mistry et al., Pfam: The protein families database in 2021. Nucleic Acids Res 49, D412D419 (2021).

95. F. Madeira et al., The EMBL-EBI search and sequence analysis tools APIs in 2019. Nucleic Acids Res 47, W636-W641 (2019).

96. T. J. Wheeler, J. Clements, R. D. Finn, Skylign: a tool for creating informative, interactive logos representing sequence alignments and profile hidden Markov models. BMC Bioinformatics 15, 7 (2014).

97. K. P. Byrne, K. H. Wolfe, The Yeast Gene Order Browser: combining curated homology and syntenic context reveals gene fate in polyploid species. Genome Res 15, 1456-1461 (2005).

98. S. Moretti et al., The M-Coffee web server: a meta-method for computing multiple sequence alignments by combining alternative alignment methods. Nucleic Acids Res 35, W645-648 (2007).

99. L. T. Nguyen, H. A. Schmidt, A. von Haeseler, B. Q. Minh, IQ-TREE: a fast and effective stochastic algorithm for estimating maximum-likelihood phylogenies. Mol Biol Evol 32, 268-274 (2015).

100. D. T. Hoang, O. Chernomor, A. von Haeseler, B. Q. Minh, L. S. Vinh, UFBoot2: Improving the Ultrafast Bootstrap Approximation. Mol Biol Evol 35, 518-522 (2018). 
Acknowledgments: We like to thank Sagie Brodsky, Tamar Jana and Offir Lupo for their strains and all Barkai lab members for fruitful scientific discussions and careful reading of the manuscript.

Funding: This work was funded by the Israel Science Foundation, ERC and the Minerva Foundation.

\section{Author contributions:}

Conceptualization: NB, TG; Methodology: TG, FJ, NB; Investigation: TG, RM; Visualization: TG, FJ; Formal analysis: TG, FJ; Data curation and Software: TG, FJ; Funding acquisition: NB; Supervision: NB; Writing - original draft: NB, TG, FJ.

Competing interests: The authors declare no competing interests.

Data and materials availability: All yeast strains are available upon request, raw and processed data is available at GEO (GSE179430). MATLAB scripts for analysis and visualization are available upon request.

\section{Supplementary Materials}

Materials and Methods

Figs. S1 to S6

Tables S1 to S4

References (77-100) 
bioRxiv preprint doi: https://doi.org/10.1101/2021.07.27.453962; this version posted August 20, 2021. The copyright holder for this preprint (which was not certified by peer review) is the author/funder, who has granted bioRxiv a license to display the preprint in perpetuity. It is made available under aCC-BY-NC-ND 4.0 International license.
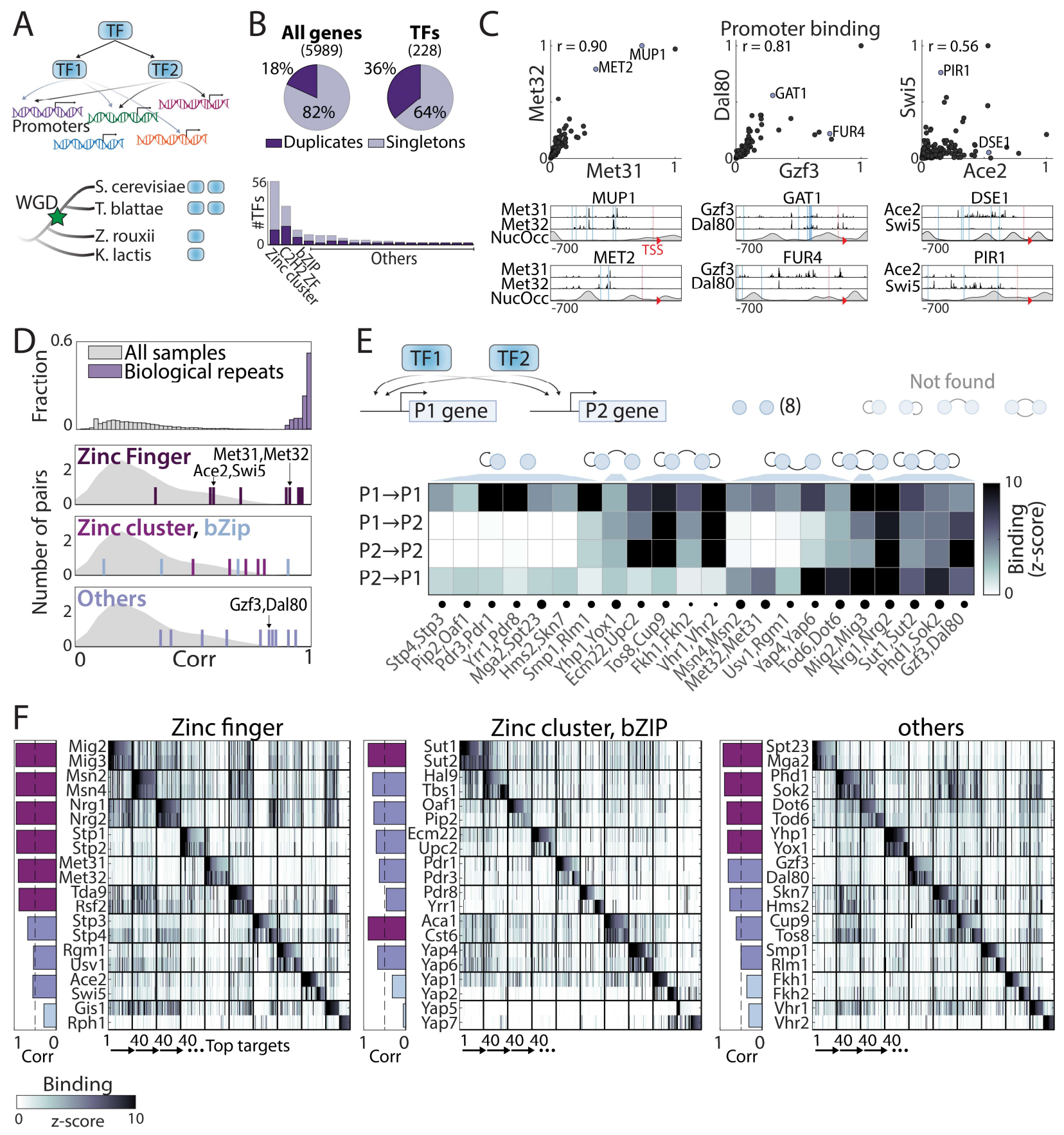


\section{Fig. 1. Promoter binding preferences of WGD TF-duplicates.}

(A-B) Whole-genome duplication (WGD) shaped the budding yeast transcription network: (A) TF duplicates (paralogs) can diverge to bind different targets. (B) In S. cerevisiae, $\sim 35 \%$ (82) of all present day TFs are retained WGD paralogs (see fig. S1). (C-D) Binding profiles of TF paralogs: Shown in (C) are promoter binding preferences of the indicated TFs (top, each dot is a promoter, r: Pearson's correlation). Bottom plots show binding signal over individual promoters, with lines indicating transcription start site (TSS, red dashed) and locations of in-vitro motifs (blue). Nucleosome positioning shown in grey. (D) Distributions of correlations between promoter binding preferences of different TFs (grey), between biological repeats (purple, top) and between paralogs (colored lines, bottom). (E) Auto- and cross-promoter binding by TF paralogs: Promoter binding signal is shown as z-score, with potentially formed circuits shown on top. Note that 22/30 pairs form six of nine possible circuits. (F) Binding to top-scoring promoters: The 40 top-bound promoters by each TF pair were selected (see table S2), ordered along the x-axis, and color-coded according to binding strength by each TF (y-axis). Bars on the left show Pearson's correlations of binding preferences between the indicated pairs. 

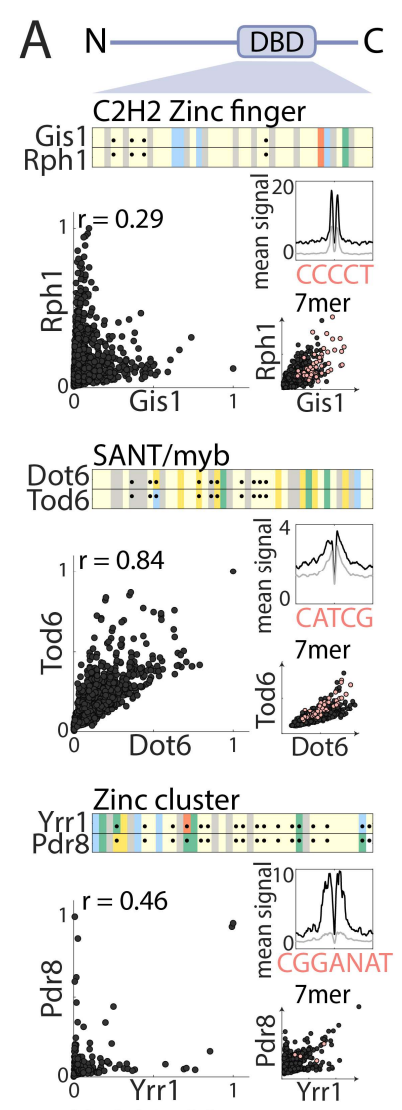

- Variable AA

- Family-defining AA

प्र० Specificity-conferring AA
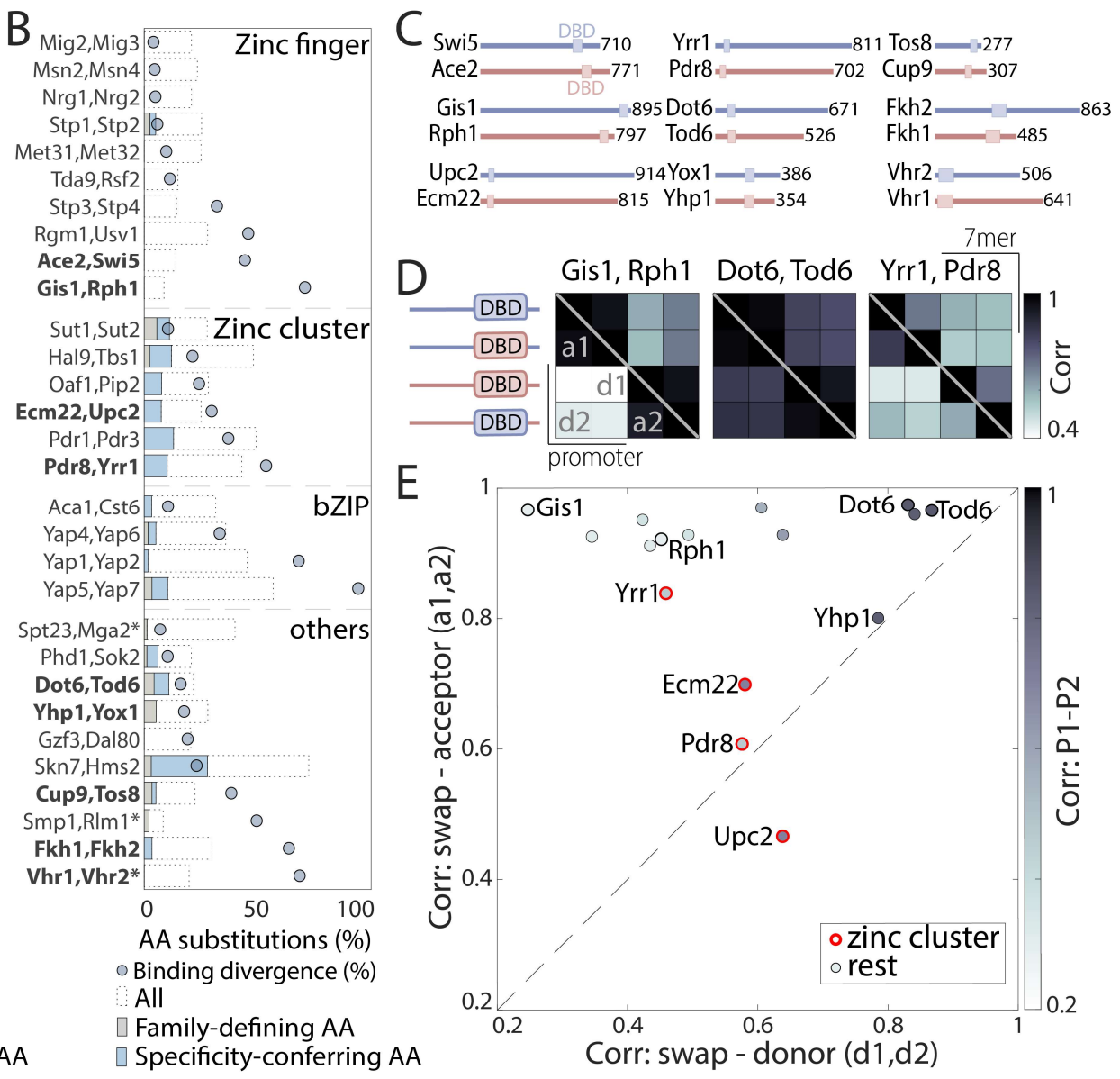


\section{Fig. 2. The contribution of DBDs to the divergence of TF paralogs.}

(A-B) Sequence variations between paralogs' DBDs: For all pairs, Pfam-defined DBDs were aligned and residues classified into those conserved among all family members (grey) and specificity-conferring ones (colored; blue, red, yellow and green denoting basic, acidic hydrophobic and hydrophilic residues respectively, (54)), as shown in (A) for the three examples (black dots indicate positions varying between paralogs). The fraction and class of varying residues is summarized in (B) (*:specificity-conferring residues not defined). Note the little correspondence between DBD sequence variations and binding profile divergence (blue dots). Paralogs chosen for further analysis are highlighted in bold. Also shown in (A) are the average signal around in-vitromotif occurrences (right, top), the respective binding signals across promoters (left) and at all 7mer sequences (right bottom, red dots indicate 7-mers containing the in-vitro motif). (C-E) DBD swapping has a minor effect on binding preferences: DBDs of the indicated paralog pairs $(\mathrm{C}$, protein length indicated) were swapped, and their binding profiles mapped. Shown in (D) are the correlations between binding preferences of the indicated TFs and their swapped variants (bottom triangle promoters, top triangles 7-mers; see also fig. S2). These correlations are summarized in (E) for all tested pairs (red indicates zinc-cluster TFs; shading depicts correlation between wild type paralogs). Note that outside the zinc-cluster family, DBD swapping is of little consequence for promoter binding preferences, even among highly divergent paralogs. Within the zinc-cluster family, DBD swapping affected binding profiles, but did not recover donor profiles. 

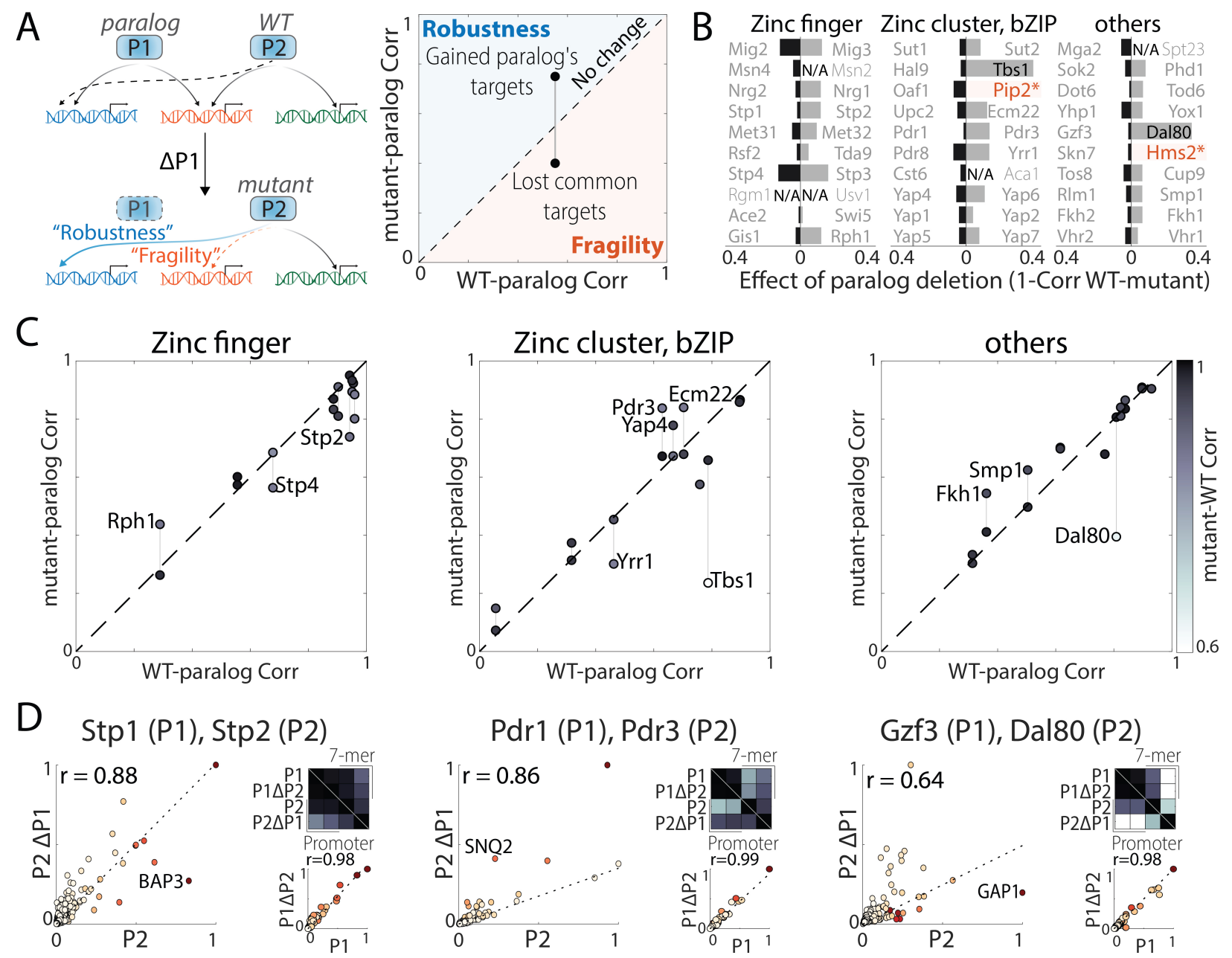

\section{$\Delta$ Binding after paralog deletion (top targets)}
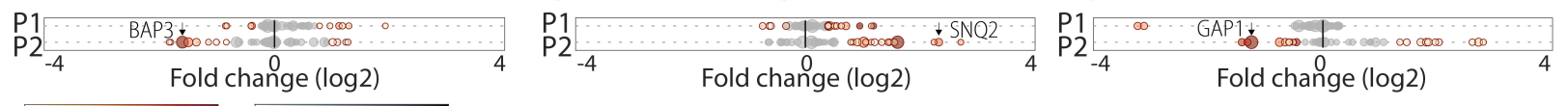

0 Paralog signal 0.80 .5 Corr

\section{Promoter signal}
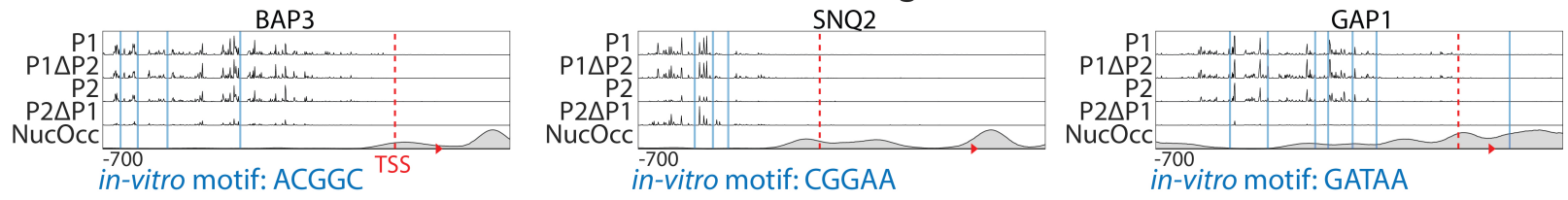


\section{Fig. 3. Interactions between TF paralogs may increase network fragility.}

(A) Paralogs' contribution to mutation robustness or fragility: Following paralog deletion, a TF may gain access to its paralog's unique sites, potentially compensating for the loss ("robustness", blue line). Alternatively, paralogs may become interdependent ("Fragility", orange line). These interactions can be visualized by comparing the TF's binding preferences in wild type (x-axis) or paralog-deleted (y-axis) backgrounds to those of the paralog. (B) Paralog interactions are rare: the effect of paralog deletion on promoter binding preferences was measured for 55 TFs of 60 paralogs in our dataset. Shown is the effect of paralog deletion on binding preference for each TF. Note that most deletions were of little effect, or asymmetric in cases of large effects. Also indicated are substantial effects (TFs highlighted in black), and TFs that completely lost binding specificity (colored in orange, N/A: not profiled, see materials and methods). (C-D) Tendency for paralog interactions varies between TF families: (C) robustness/fragility analysis, as in (A) for all tested paralog pairs, divided into families. (D) For individual examples, binding is shown over all (top) or individual promoters (bottom), and as relative change following paralog deletion (middle; dot color and size indicate a TF's and its paralog's binding signal, respectively). Note that Stp2 and Dal80 lose binding to some of the targets shared with their respective paralog following paralog deletion ("fragility"), whereas Pdr3 gains binding to Pdr1 targets (SNQ2) upon the latter's deletion ("robustness"). 


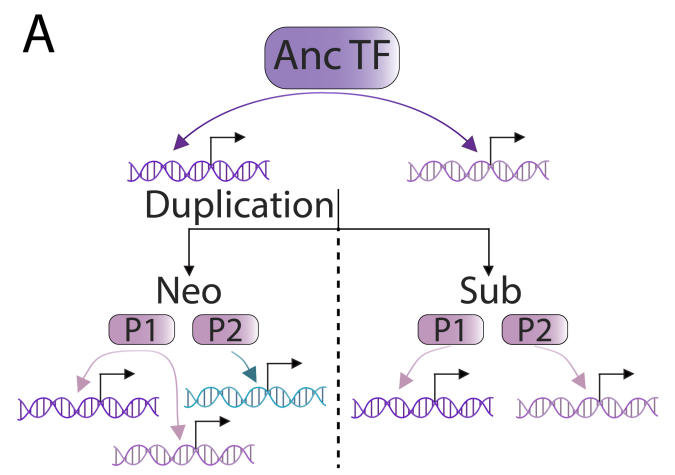

B non-DBD phylogeny
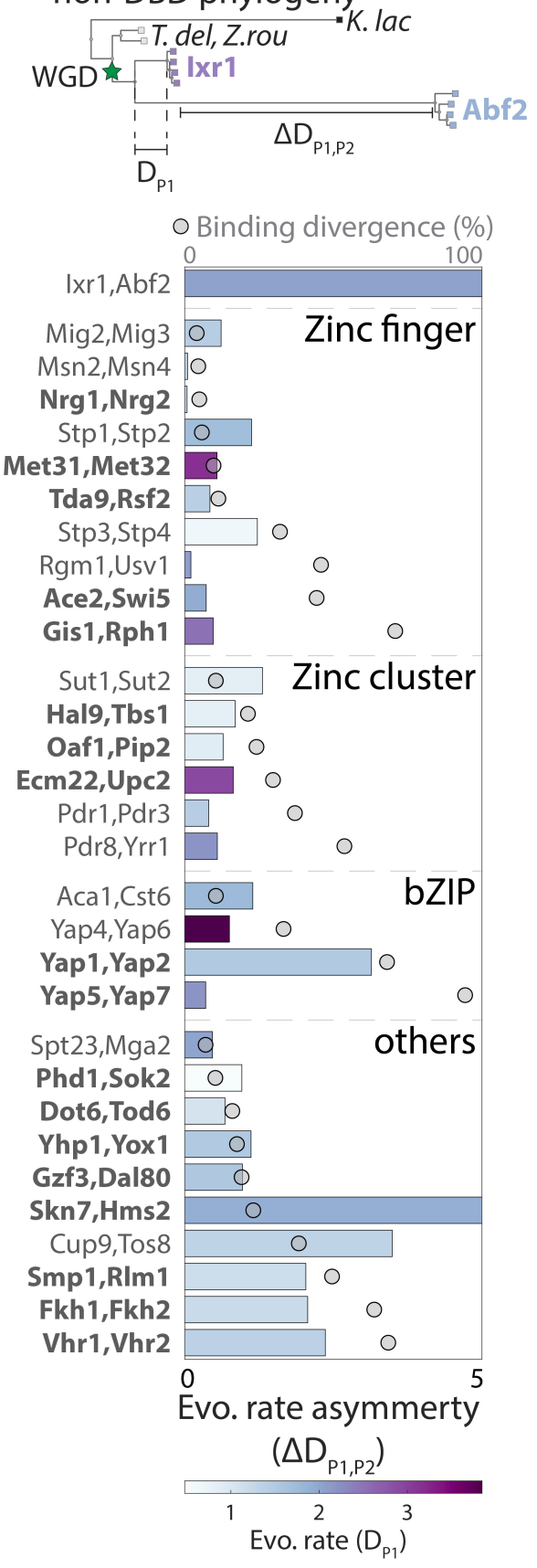
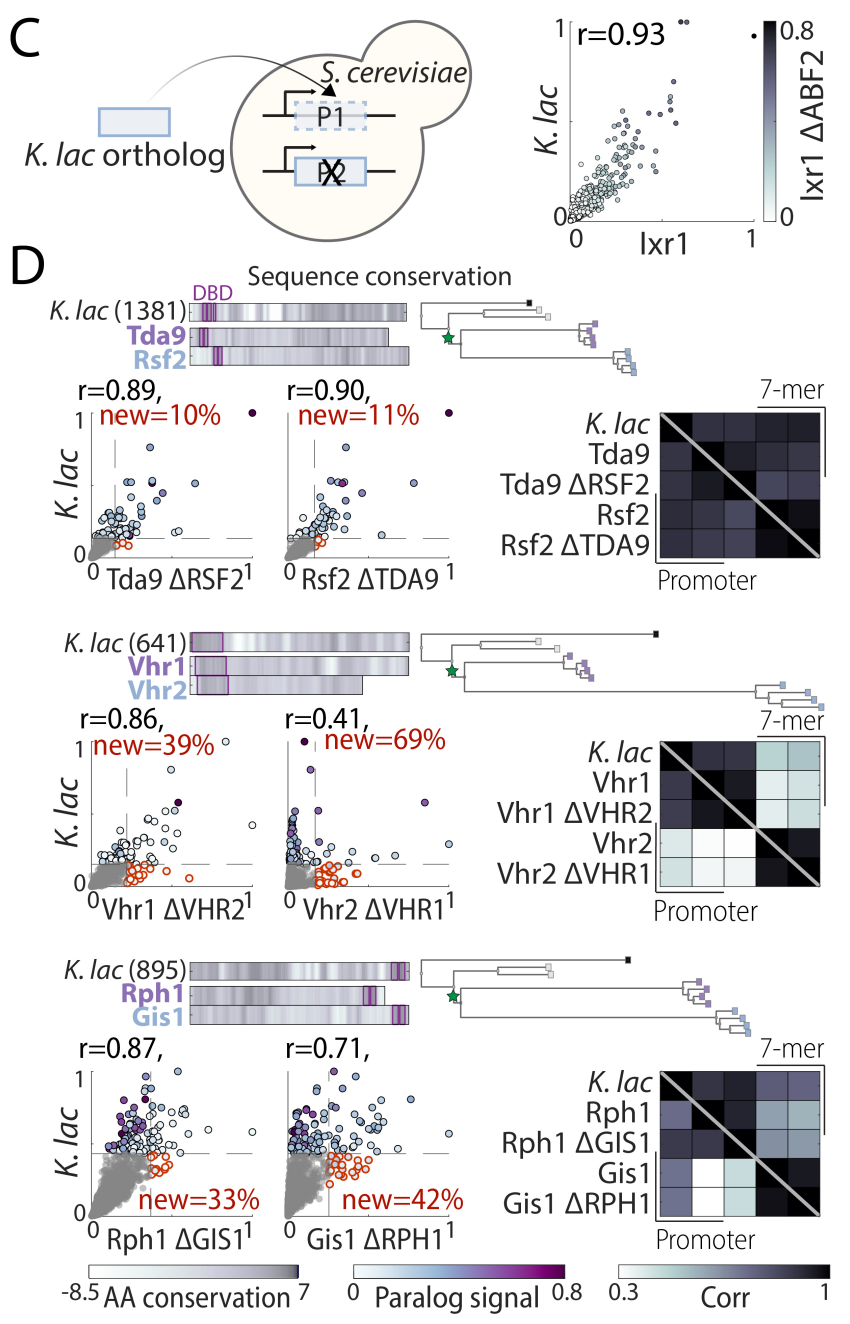

$\mathrm{E}$

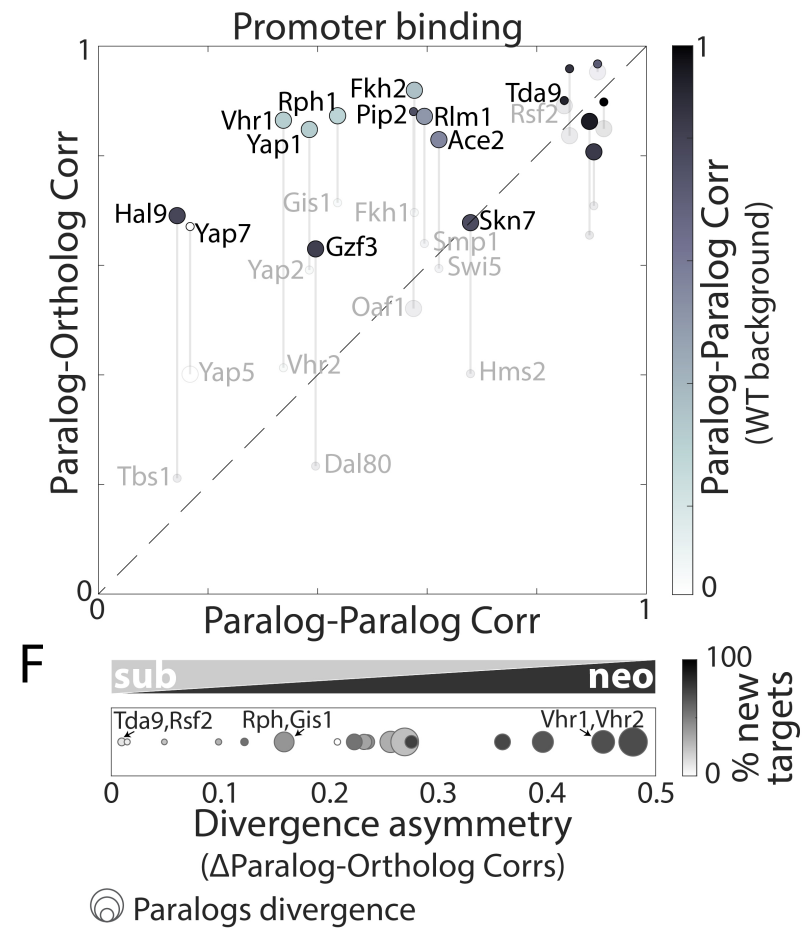




\section{Fig.4. Classifying paralog divergence through comparison to non-WGD orthologs.}

(A) Models of evolutionary paths: Paralogs could diverge by acquiring new preferences (neofunctionalization) or by splitting ancestral preferences (sub-functionalization). (B) Sequence evolution: Top: Phylogenetic comparison of non-DBD sequences of the Ixr1/Abf2 paralogs, indicating distances from last common ancestor (LCA). Bottom: differences in sequence evolution rate between paralogs (evolutionary rate asymmetry, $\Delta \mathrm{D}_{\mathrm{P} 1, \mathrm{P} 2}$, see methods for details). Color indicates rate of the conserved paralog $\left(\mathrm{D}_{\mathrm{P} 1}\right)$, and grey dots indicate divergence of promoter binding preferences. Paralogs chosen for further analysis are highlighted in bold. (C-F) Binding preferences of non-WGD orthologs: Binding profiles of $K$. lactis orthologs were mapped within S. cerevisiae and compared to both paralogs (left). Ixr1 binding is tightly conserved between $S$. cerevisiae and $K$. lactis (right). Shown are the correlations of binding preference between the $K$. lactis and S. cerevisiae orthologs for specific examples (D) and for all tested paralogs (E; bigger spot size corresponds to the paralog with slower sequence evolution; see also fig. S4). Note the high similarity of binding preferences between each $K$. lactis TF with at least one of its $S$. cerevisiae paralogs, commonly the one experiencing slower sequence divergence. Divergence asymmetry is quantified in $(\mathrm{F})$, with colors indicating the fraction of 'new' targets acquired by the less conserved paralog. 
bioRxiv preprint doi: https://doi.org/10.1101/2021.07 27.453962; this version posted August 20, 2021. The copyright holder for this preprint (which was not certified by peer review) is the author/funder, who has granted bioRxiv a license to display the preprint in perpetuity. It is made available under aCC-BY-NC-ND 4.0 International license.

A

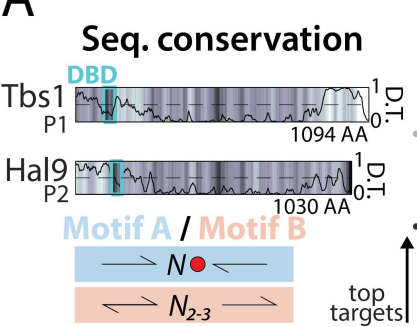

$\underset{(w t)}{\text { Binding }}$ Motif Binding change $\quad$ score $(\Delta$ Paralog/wt)

Promoter Signal
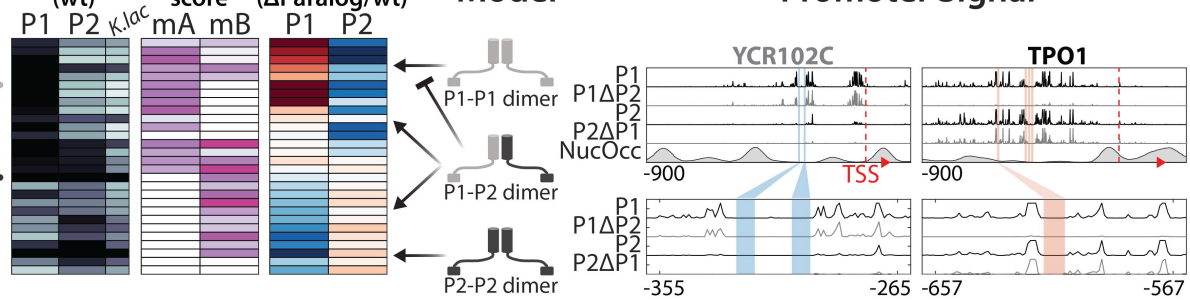

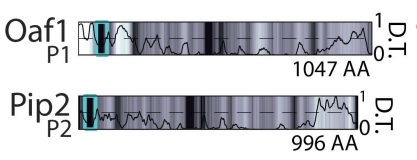

$\longrightarrow N_{2-5}$
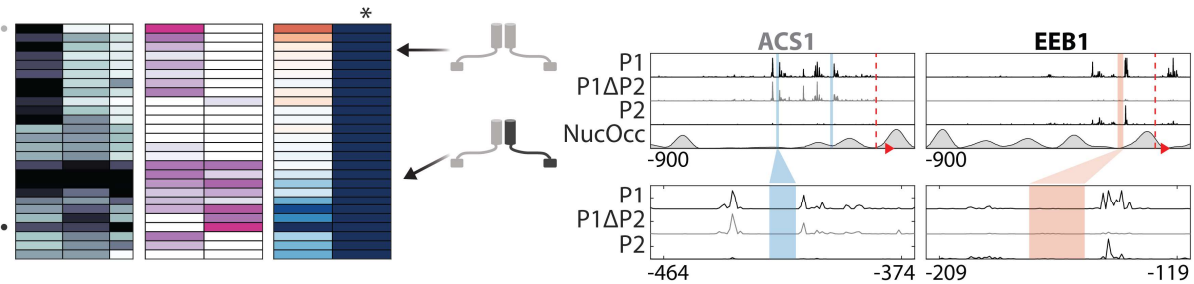

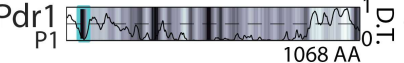

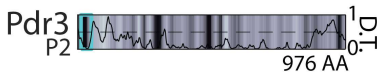
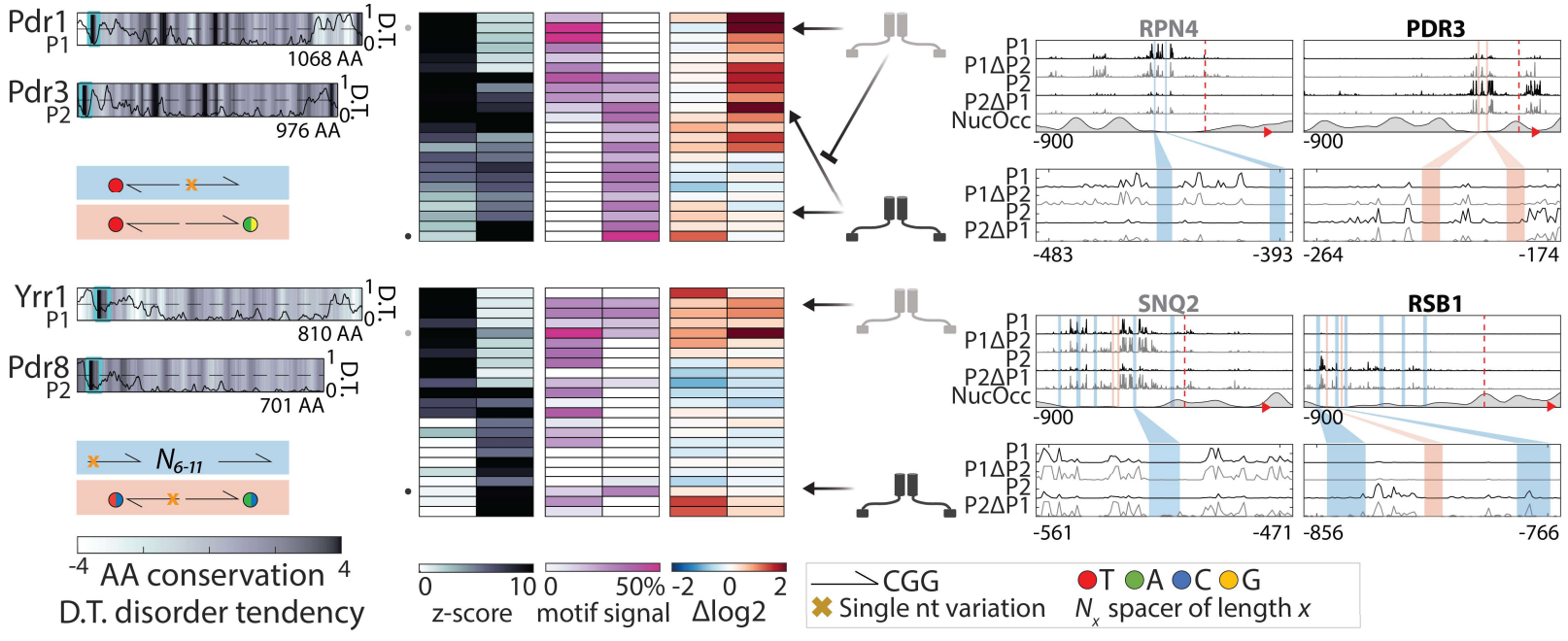

z-score motif signal $\Delta \log 2 *$ Single nt variation $N_{x}$ spacer of length $x$

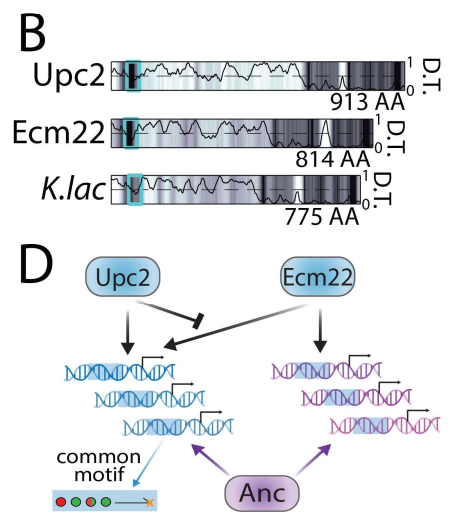

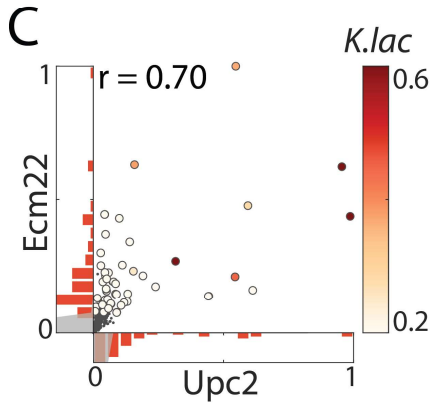

K.lac top targets all genes
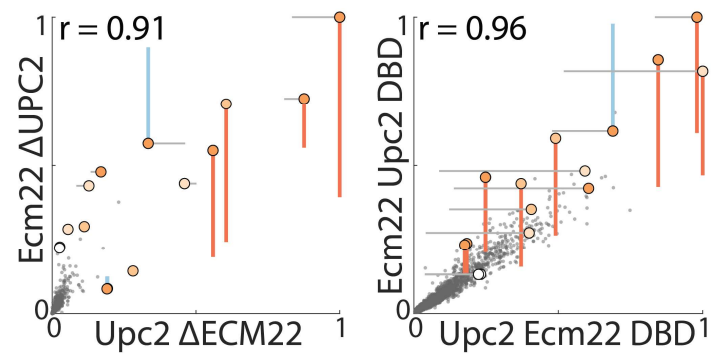

- mutant $>$ WT \begin{tabular}{|} 
Number of motif occurrences \\
\begin{tabular}{|cccc}
\hline 0 & 1 & 2 & $>2$
\end{tabular}
\end{tabular} 


\section{Fig. 5. Divergence of zinc-cluster TF paralogs correlates with changes in motif preferences.}

(A) Dimerization and changes in motif-preferences may explain divergence of zinc-cluster paralogs: Zinc-cluster paralogs vary in sequence and localized at different variants of their characteristic motif, shown on the left (AA sequence conservation in color-code, DBD indicated as cyan box, and disorder tendency (76) shown as black line; motif symbols indicated on the bottom, see fig. S5 for motif sequences). For each pair, top-bound promoters were selected, and peak-proximal motifs defined. Shown, as indicated, are promoter binding (z-score), percentage of total promoter signal 50 bases around the indicated motifs, and binding change upon paralog deletion ( $\log 2)$. Suggested models explaining divergence and signal on exemplary promoters (indicated by small dots next to the binding panel) are also shown. (B-D) Upc2/Ecm22 diverge through DNA-binding competition: shown in (B) is the disorder tendency and pair-wise sequence conservation of Upc2-Ecm22 and the K. lactis ortholog with Upc2. Promoter binding preferences in the indicated backgrounds are shown in (C). Large dots indicate top $50 \mathrm{~K}$. lactis targets, colorcoded by binding strength. Distribution of these targets across the Upc2/Ecm 22 binding preference are shown as histograms (red, grey: all genes). Note that Upc2 and Ecm22 bind comparably to strong $K$. lactis targets, while Ecm 22 dominates on the low-intermediate targets. Colors in mid/right panels indicate the number of occurrences of the known in-vitro motif (TA(T/A)ACGA) and lines show change in binding relative to the wild type. A suggested model summarizing these results is depicted in (D). 
bioRxiv preprint doi: https://doi.org/10.1101/2021.07 27.453962; this version posted August 20, 2021. The copyright holder for this preprint (which was not certified by peer review) is the author/funder, who has granted bioRxiv a license to display the preprint in perpetuity. It is made available under aCC-BY-NC-ND 4.0 International license.
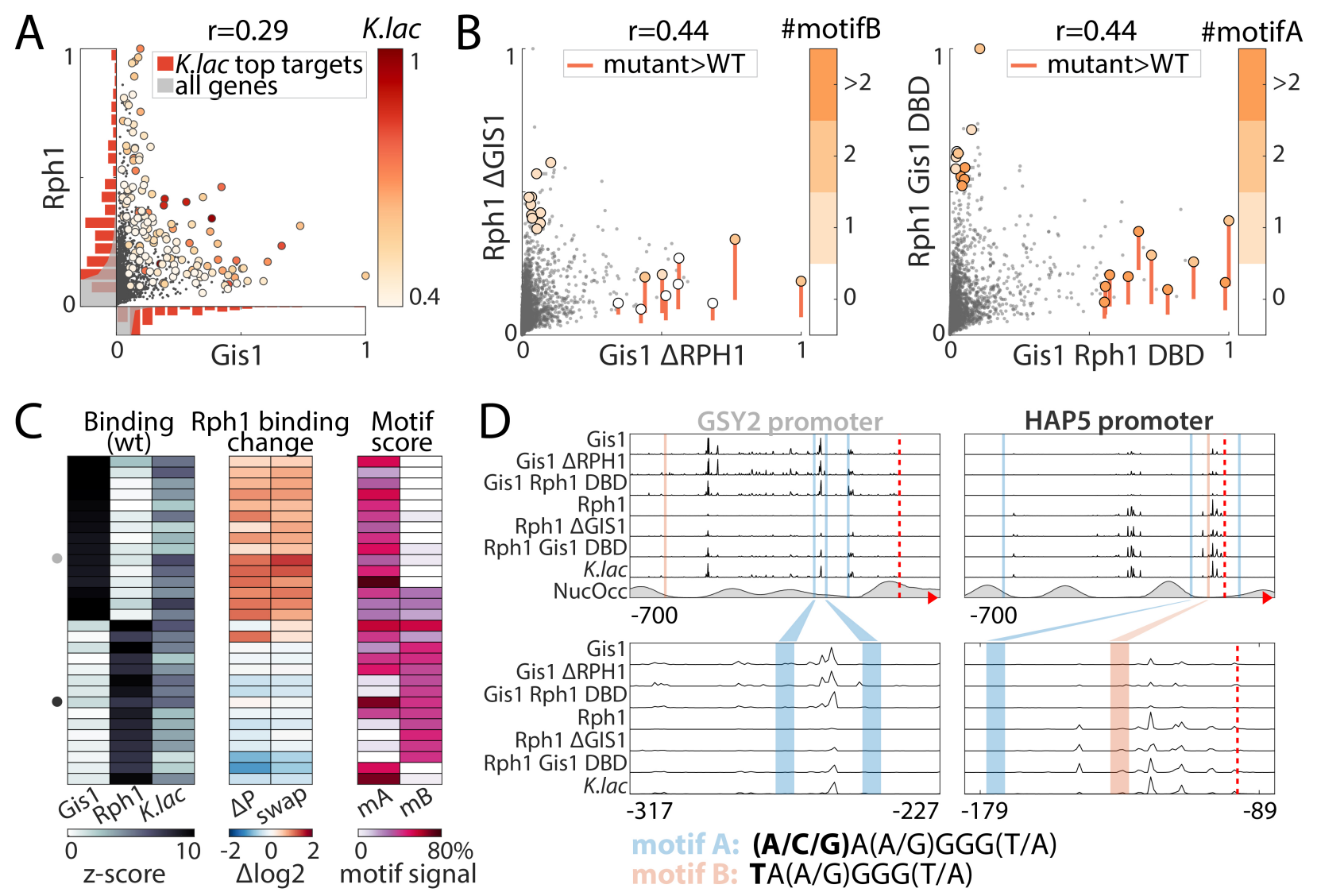

$\mathrm{E}$

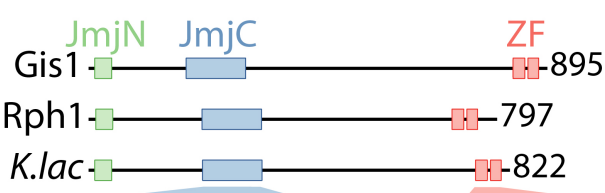

Gis1 282 ...EFIITFPYCMYSGFNYGYN . . 300 832 ...RECNRQFSSGH . . 842

Rph1 313...EFMITYPYGYHAGFNYGYN...331 713...KECQRKFSSGH . . 723

K. lac $314 \ldots$. EFIVTYPFGYHAGFNYGYN . . 332 759... QECARQFSSGH . . 769
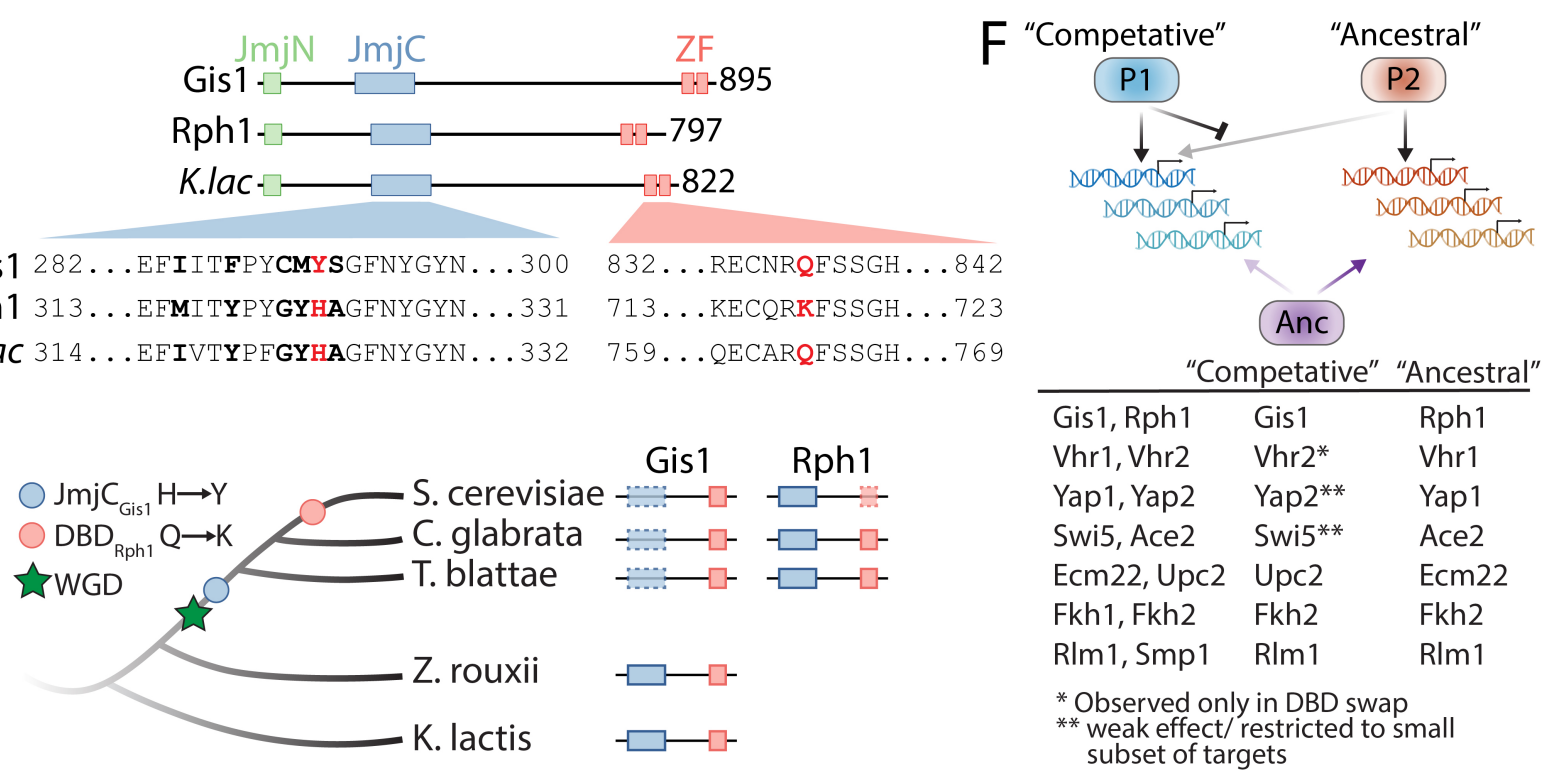

\begin{tabular}{lll}
\hline Gis1, Rph1 & Gis1 & Rph1 \\
Vhr1, Vhr2 & Vhr2* & Vhr1 \\
Yap1, Yap2 & Yap2** & Yap1 \\
Swi5, Ace2 & Swi5** $^{* *}$ & Ace2 \\
Ecm22, Upc2 & Upc2 & Ecm22 \\
Fkh1, Fkh2 & Fkh2 & Fkh2 \\
Rlm1, Smp1 & Rlm1 & RIm1 \\
* Observed only in DBD swap \\
** weak effect/ restricted to small \\
subset of targets
\end{tabular}




\section{Fig. 6. Resolution of paralog interference through competitive binding.}

(A-D) Gis1 limits Rph1 binding through DBD-dependent binding competition: shown are promoter binding preferences of Gis1 and Rph1 in wild type backgrounds (A, as in Fig. 5C) and following mutual paralog-deletion and DBD swapping $(\mathrm{B}$, colored by the number of occurrences of the two known motif variants specified in D). The analysis of all top-bound promoters is summarized in $(C)$ and binding signals on exemplary promoters are shown (D, as described in Fig. 5A). Note the increased binding of Rph1 to Gis1 target promoters upon GIS1 deletion or DBD swapping (e.g. GSY2), and reduced Gis1 binding to its target promoter after DBD swapping (e.g. HAP5). (E) Gis1's loss of demethylase activity preceded variation in Rph1's DBD: The conserved JmjC domain providing Rph1 a histone demethylase activity is mutated in Gis 1 orthologs of all post-WGD species. The respective DBDs differ in only four positions, one of which replaced a conserved glutamine by lysine specifically in Rph1 and its closest orthologs (fig. S6). This suggests that the divergence was triggered by the loss of demethylase function and DBDindependent acquisition of new targets by Gis1, and finally mutation in Rph1-DBD to reduce residual Rph1 binding interference at the newly acquired Gis1 sites. (F) Resolution of paralog interference among diverging TF paralogs: A model for the resolution of paralog interference through competitive binding. The TF inhibiting its paralog's binding is denoted as 'competitive', while the TF whose binding preference better resembles that of the K. lactis ortholog is denoted as 'ancestral'. In addition to Gis1/Rph1, other diverging paralogs whose K. lactis orthologs were profiled appear to conform to this general model (fig. S6). Note that in most cases (indicated), divergence in promoter binding is driven by variations outside the DBD, with competition only refining, but not driving this divergence of target preferences. 